\title{
Association between the BRAF V600E mutation and ultrasound features of the thyroid in thyroid papillary carcinoma
}

\author{
QIAN LI ${ }^{1,2}$, JIANJUN YUAN $^{1}$, YAN WANG $^{2}$ and YUANPENG ZHAI ${ }^{2}$ \\ ${ }^{1}$ Department of Medical Ultrasonics, Henan Provincial People's Hospital, People's Hospital of \\ Zhengzhou University, Zhengzhou, Henan 450003; ${ }^{2}$ Department of Medical Ultrasonics, Affiliated \\ Cancer Hospital of Zhengzhou University, Zhengzhou, Henan 450008, P.R. China
}

Received January 17, 2017; Accepted May 12, 2017

DOI: $10.3892 / \mathrm{ol} .2017 .6276$

\begin{abstract}
The aim of the present study was to investigate the association between the BRAF V600E mutation and ultrasound features of the thyroid in papillary thyroid carcinoma (PTC). Fresh thyroid carcinoma tissue and paracarcinoma tissue were obtained from 34 patients undergoing surgery for PTC. The BRAF V600E mutation was detected by PCR amplification and direct DNA sequencing. The thyroid ultrasound results were compared between patients with and without the BRAF V600E mutation. Eighteen out of 34 cases were identified with the BRAF V600E mutation (52.9\%), while 16 cases did not have the BRAF V600E mutation (47.1\%). Additionally, no BRAF V600E mutation was detected in paracarcinoma tissue in the 34 cases. The results of ultrasound imaging suggested that there were no significant differences in tumor size, whether the border was clear, or in tumor calcification (presence or absence) between patients with and without BRAF V600E mutation $(\mathrm{P}>0.05)$. The BRAF V600E mutation rate was high in patients with PTC. There was no significant correlation between BRAF V600E mutation and thyroid ultrasound features. Thyroid ultrasound features are therefore unable to predict the presence of the BRAF V600E mutation in patients with PTC.
\end{abstract}

\section{Introduction}

Thyroid carcinoma is a common endocrine malignancy. It accounts for $1.3 \%$ of systemic malignancies and $5.1 \%$ of head and neck cancer. Thyroid cancer has a variety of pathological types, and the most common type is papillary thyroid carcinoma (PTC), which accounts for 59.5-89.0\% of thyroid carcinoma (1). At present, it is commonly believed that the occurrence and development of cancer are closely related to

Correspondence to: Dr Jianjun Yuan, Department of Medical Ultrasonics, Henan Provincial People's Hospital, People's Hospital of Zhengzhou University, 7 Weiwu Road, Zhengzhou, Henan 450003, P.R. China

E-mail: yph65n@163.com

Key words: papillary thyroid carcinoma, BRAF V600E, ultrasound changes at the genetic level. Various genetic techniques have been applied for the diagnosis and treatment of tumors. Several genes have been identified that are related to the occurrence, development, and prognosis of thyroid cancer (2). In recent years, genetic testing of thyroid benign and malignant tumors has been extensively performed, and the results suggest there is a high frequency of BRAF mutations in PCT. By contrast, BRAF mutations have not been detected in any other benign or malignant thyroid tumors (1-3). This indicates that BRAF mutations are important genetic events in PTC, and are closely related to its occurrence and development. BRAF, also known as murine sarcoma viral oncogene homolog B1, is a member of the RAF gene family (4). At present, over 30 types of BRAF mutations have been identified, of which approximately $90 \%$ are detected in exon 15, and 10\% in exon 11 (4). An important BRAF mutation in PTC is thymine substituted with adenine at nucleotide 1799 of the 11th and 15th exons, which results in a missense mutation of codon 600 (V600E) $(4,5)$. This leads to valine being substituted by glutamate. It has been reported that the frequency of the BRAF V600E mutation varies greatly (28-83\%) (5-8).

Thyroid B ultrasound is a form of ultrasound examination. The principle of imaging is based on the differences of reflection, absorption, and attenuation of ultrasonic waves in thyroid tissue and surrounding neck tissues. Thyroid B ultrasound can clearly show the anatomical morphology of the thyroid, its size, internal tissue echo, the presence or absence of nodules, and tumors. In addition, it can detect deep thyroid nodules which are undetectable by common physical examination. Therefore, it is a preferred auxiliary examination for patients with thyroid diseases (9-13), and a preferred method for the evaluation of anatomic abnormalities of the thyroid and for the guidance of fine needle aspiration biopsy. High frequency and high resolution ultrasound probes, as well as color Doppler ultrasound further improve the accuracy of ultrasound diagnosis. Some features of ultrasound such as low echo, microcalcification, and increased blood flow in nodules detected by Doppler ultrasound are important for the diagnosis of malignant nodules and thyroid cancer (6). However, the association between the BRAF V600E mutation and thyroid ultrasound features in patients with PTC remains unclear.

The aim of the present study was to investigate the incidence of BRAF mutations in patients with PTC, by analyzing BRAF 
mutations in fresh thyroid carcinoma tissue and paracarcinoma tissue, and to compare the differences in PTC patients with and without BRAF V600E mutations, thus providing a basis for the clinical diagnosis and treatment of thyroid cancer.

\section{Materials and methods}

General information. Fresh thyroid carcinoma tissue and paracarcinoma tissue (over $5 \mathrm{~cm}$ away from the edge of carcinoma tissue) were collected from 34 patients undergoing surgery for PTC from December 2009 to June 2010 in People's Hospital of Zhengzhou University (Henan, China). Thyroid carcinoma was confirmed by histopathological analysis. The 34 patients included 28 females and 6 males, aged 16-75 years. The thyroid ultrasound features of patients were obtained using GE Logiq 9 and GE Logiq 700 (both from GE Healthcare, Piscataway, NJ, USA) color Doppler ultrasound scanners (frequency: 10-14 $\mathrm{MHz}$ ). For each patient, tumor size (recorded at its largest diameter), border (clear or unclear), and the presence or absence of calcifications were analyzed. This study was approved by the Ethics Committee of Henan Provincial People's Hospital. Signed written informed consents were obtained from all participants before the study.

Reagents. Tissue lysis solution, protease K solution (20 mg/ml), phenol:chloroform:isoamyl alcohol (25:24:1), isoamyl alcohol, chloroform, $3 \mathrm{M}$ sodium acetate solution, $75 \%$ ethanol, and double-distilled water were purchased from Guangzhou Chemical Reagent Factory (Guangzhou, China). Taq enzymes and related reagents were purchased from Tiangen Biotech Co., Ltd. (Beijing, China). Protein molecular weight marker and PCR marker were purchased from Takara Bio, Inc. (Otsu, Japan). Agarose powder was purchased from the Shanghai Institute of Medicine of Chinese Academy of Medical Sciences (Shanghai, China). TBE buffer, shrimp alkaline phosphatase, and exonuclease were purchased from Zhong Shan Golden Bridge Biotechnology Co., Ltd. (Beijing, China).

Instruments and equipment. Instruments used in the present study were: Horizontal agarose electrophoresis equipment (Shanghai Jingyi Organic Glass Products and Instruments Factory, Shanghai, China), centrifuge, $37^{\circ} \mathrm{C}$ incubator (Thermo Fisher Scientific, Waltham, MA, USA), $-20^{\circ} \mathrm{C}$ refrigerator (Qingdao Haier Pharmaceutical Co., Ltd., Qingdao, China), DNA thermal cycler 480 (Perkin-Elmer, Norwalk, CT, USA), ABI 3730 DNA Sequencer (Perkin-Elmer Applied Biosystems, Foster City, CA, USA); PTC-200 high throughput PCR machine (MJ Research, Inc., South San Francisco, CA, USA); and ultraviolet spectrophotometer (Mettler-Toledo, Schwerzenbach, Switzerland).

Extraction of genomic DNA. Thyroid tissue was placed in a $2 \mathrm{ml}$ centrifuge tube. Tissue lysis buffer $(800 \mu \mathrm{l})$ and $20 \mu \mathrm{l}$ of pre-cooled proteinase $\mathrm{K}$ solution were added. Centrifuge tubes were placed on a shaker for gentle agitation (100-150 times $/ \mathrm{min})$ at $50^{\circ} \mathrm{C}$ overnight. A total of $820 \mu \mathrm{l}$ of phenol:chloroform:isoamyl alcohol (25:24:1) was added and then gently mixed for 10 min until the solution turned milky-white. The samples were centrifuged at $188.942 \mathrm{x}$ g for $15 \mathrm{~min}$ at $4^{\circ} \mathrm{C}$. The supernatant was aspirated and transferred

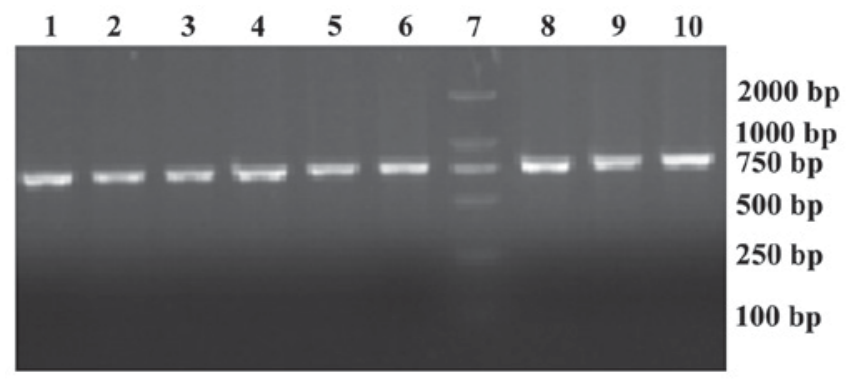

Figure 1. Electrophoresis results of PCR reaction products. Part of exon 15 of BRAF was amplified using specific primers. The 7th band is the marker (range: 500-2,000 bp), and the remaining bands are the PCR products of nine samples. Products were between 750 and $1,000 \mathrm{bp}$

to another centrifuge tube. An equal volume of chloroform was then added to the supernatant, mixed gently for $10 \mathrm{~min}$, and centrifuged at $11,500 \mathrm{x}$ for $15 \mathrm{~min}$ at $4^{\circ} \mathrm{C}$. The supernatant was transferred to another $2 \mathrm{ml}$ centrifuge tube. An equal volume of pre-cooled $\left(-20^{\circ} \mathrm{C}\right)$ isopropanol and $10 \%$ of the supernatant volume of pre-cooled $\left(0^{\circ} \mathrm{C}\right)$ sodium acetate were added, and gently mixed by inversion 10 times until the flocculation precipitate was observed. The samples were then centrifuged at $11,500 \mathrm{x}$ g for $5 \mathrm{~min}$ at $4^{\circ} \mathrm{C}$. The supernatant was discarded, $1 \mathrm{ml}$ of $75 \%$ ethanol was added, and the samples were centrifuged at $11,500 \mathrm{x} \mathrm{g}$ and $4^{\circ} \mathrm{C}$ for $5 \mathrm{~min}$. Ethanol was removed, and the precipitate was washed once with $75 \%$ ethanol. Ethanol was discarded and samples were centrifuged at $11,500 \mathrm{x}$ for $5 \mathrm{~min}$ at $4^{\circ} \mathrm{C}$. The remaining ethanol was aspirated, and the precipitate was left to air dry for 20-30 min until it became semi-translucent. Next, 30-50 $\mu 1$ of double-distilled water was added to dissolve the sample. Samples were placed at $37^{\circ} \mathrm{C}$ for $30 \mathrm{~min}$ for better dissolution, then cooled and stored at $-20^{\circ} \mathrm{C}$; or placed at $4^{\circ} \mathrm{C}$ overnight until DNA was completely dissolved and then stored at $-20^{\circ} \mathrm{C}$.

Identification of genomic DNA and determination of concentration. After DNA was completely dissolved at room temperature, absorbance (OD value) at 260 and $280 \mathrm{~nm}$ was measured. Distilled water was used as a blank control.

Identification of DNA purity and quality. The OD 260/280 nm ratio was calculated. The purity of DNA was considered high if the ratio was close to 1.8 , while a ratio of $>2.0$ indicated RNA contamination, and ratio of $<1.6$ indicated organic solvent contamination.

Polymerase chain reaction. Primers used for amplification of the $B R A F$ gene and $B R A F$ mutant genes were designed using Primer Premier 7.0 (Primer, Quebec, Canada), and produced by the Shanghai Bioengineering Co., Ltd. (Shanghai, China). The upstream and downstream primers, respectively, were: 5'-CATCCTAACACATTTCAAGCCCC-3', and 5'-TCACAC CTGCCTTAAATTGCATAC-3'. The amplified fragment was $795 \mathrm{bp}$ in length. The volume of the PCR reaction system was $20 \mu \mathrm{l}$. The PCR cycles were performed on a PTC-200 high-throughput PCR machine. The thermal profile was as follows: pre-denaturation at $95^{\circ} \mathrm{C}$ for $5 \mathrm{~min}$ (denaturation at 


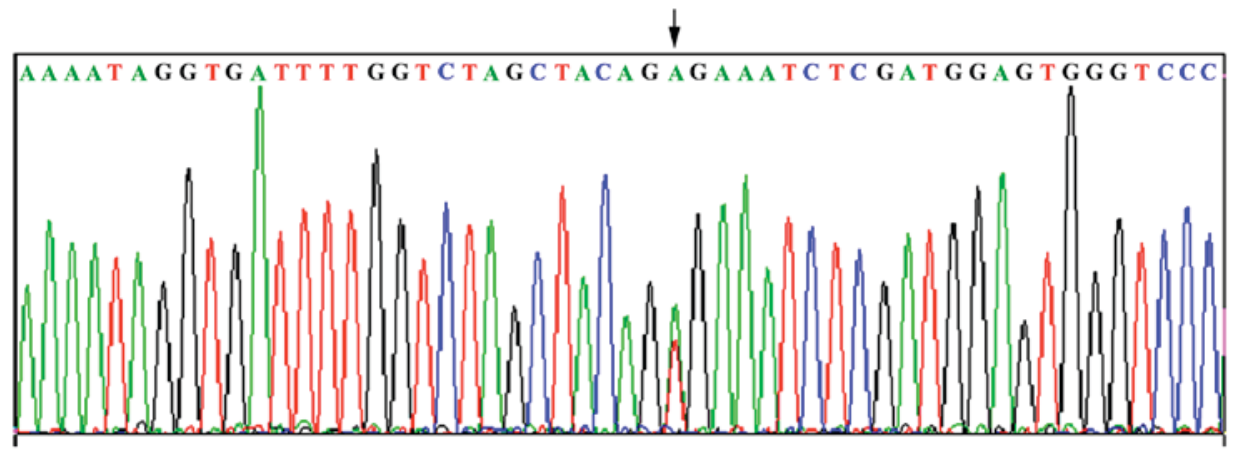

Figure 2. Direct DNA sequencing results of the carcinoma tissue with mutations ( $\downarrow$ indicates the mutation point).

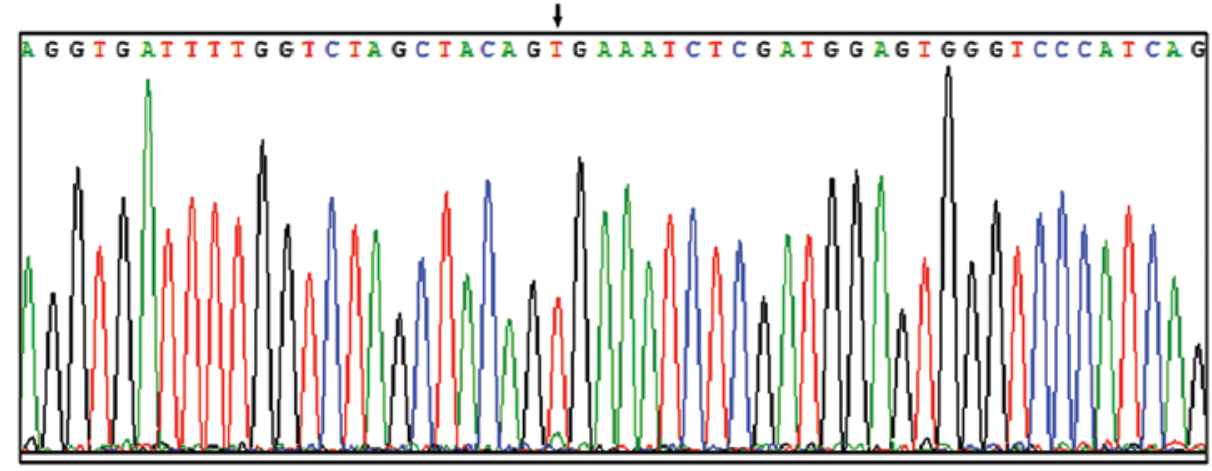

Figure 3. Direct DNA sequencing results of the carcinoma tissue without mutations ( $\downarrow$ indicates no mutation).

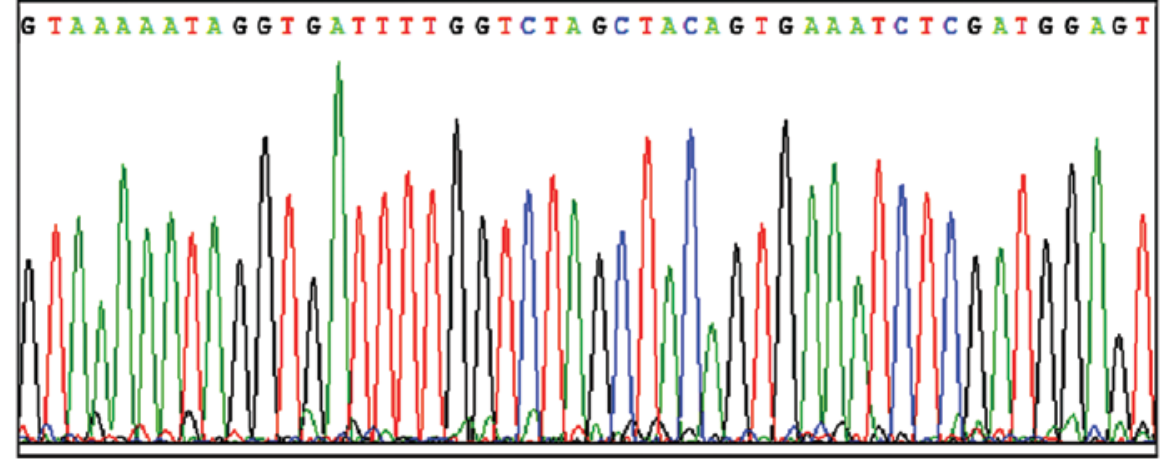

Figure 4. Direct DNA sequencing results of the paracarcinoma tissue ( $\downarrow$ indicates no mutation)

$95^{\circ} \mathrm{C}$ for $30 \mathrm{sec}$, annealing at $59^{\circ} \mathrm{C}$ for $30 \mathrm{sec}$, extension at $72^{\circ} \mathrm{C}$ for $55 \mathrm{sec}$ ) x 35 cycles, extension at $72^{\circ} \mathrm{C}$ for $10 \mathrm{~min}$.

DNA sequencing. The PCR products were sent to Shanghai Biotechnology Co., Ltd. (Shanghai, China) for sequencing to detect the BRAF V600E mutation. The sequencing results were compared using BLAST. The results included the base pairs TT, TA, and AA, which were likely present at nucleotide 1799.

Statistical analysis. Data were analyzed using SPSS 17.0 (IBM Corp., Armonk, NY, USA) software. Statistical analyses were performed using the t-test and the exact probability test. $\mathrm{P}<0.05$ was considered to indicate a statistically significant difference.

\section{Results}

Results of DNA sequencing. Part of exon 15 of BRAF was amplified using specific primers, and the length of the product was $795 \mathrm{bp}$. The results of electrophoresis of the PCR reaction products are shown in Fig. 1. The 7 th lane shows the marker (500-2,000 bp), while the remaining lanes represent the electrophoresis bands of the PCR reaction products from nine samples. All the products were between 750 and 1,000 bp. The BRAF V600E mutation was detected in 18 out of 34 cases $(52.9 \%)$, with a $\mathrm{T}$ to $\mathrm{A}$ transition at nucleotide 1799 in exon 15 (Fig. 2, two signal peaks superimposed), resulting in valine 600 being substituted by tryptophan. No BRAF V600E mutations were detected in 16 out of 34 cases (47.1\%) (Fig. 3) and all 34 paracarcinoma 
Table I. Frequency of genotype and alleles.

\begin{tabular}{lcccccc}
\hline & \multicolumn{3}{c}{ Genotype $(\%)$} & & \multicolumn{2}{c}{ Allele (\%) } \\
\cline { 2 - 3 } \cline { 6 - 7 } Tissue & $\mathrm{T} / \mathrm{T}$ & $\mathrm{T} / \mathrm{A}$ & $\mathrm{A} / \mathrm{A}$ & & $\mathrm{T}$ & $\mathrm{A}$ \\
\hline Carcinoma & $16(47.1)$ & $18(52.9)$ & $0(0)$ & $50(73.5)$ & $18(26.5)$ \\
Paracarcinoma & $34(100)$ & $0(0)$ & $0(0)$ & $68(100)$ & $0(0)$ \\
\hline
\end{tabular}

tissue samples (Fig. 4). All 18 cases with mutations were heterozygous mutations with base TA type. The genotypic and allelic frequencies in each group are presented in Table I.

Comparison of thyroid ultrasound features between patients with and without BRAF V600E mutations. The features and differences of thyroid ultrasound between patients with and without BRAF V600E mutations are shown in Table II. There were no significant differences $(\mathrm{P}>0.05)$ in tumor size, tumor border, or calcification between the BRAF V600E mutation group and the non-mutation group according to the ultrasound examination.

\section{Discussion}

BRAF, also known as murine sarcoma viral carcinogenic homology B1, was identified by Ikawa et al 28 years ago as an oncogene (4). Its biological role is to induce the proliferation of avian primary cells and the transformation of NIH3T3 cells. BRAF is a member of the RAF family of proteins. It is a specific serine/threonine kinase, and the most critical activator of MEK/ERK (8-11).

It was reported that the BRAF V600E protein induced the transformation of thyroid cells in vitro (6). Moreover, thyroid cancer was induced in transgenic mice with thyroid tissue that specifically expressed the BRAF V600E protein, suggesting that BRAF protein activation is closely related to the occurrence of PTC. The role and potential mechanism of BRAF mutation in the occurrence and development of PTC has become an area of great interest (7). It is currently believed that the V600E mutation is located in the activation region of BRAF (CR3). A negative charge induced by mutation mimics the phosphorylation of threonine 598 and serine 601, resulting in aberrant activation of BRAF, which enhances its ability to activate downstream kinases by up to 12.5 times compared with the wild-type protein. Activated BRAF then transfers signals to downstream molecules, through the RAS/RAF/MEK/ERK/MAPK pathway, promoting the proliferation of thyroid cells and tumor formation (8). Sithanandam et al (9) and Mercer and Pritchard (10) found that BRAF was the strongest activator of the MAPK pathway. When a BRAF mutant was transfected into NIH3T3 (mouse embryonic fibroblast cell line) cells, transformation efficiency was enhanced by 70 - to 138-fold compared with wild-type $(10,11)$, which further supported the observation that BRAF mutation induces carcinogenesis through the RAS/RAF/MEK/ERK/MAPK pathway. The continuous activation of the RAS/RAF/MEK/MAPK signaling pathway also promoted the change from well-differentiated papillary carcinoma cells to poorly differentiated and undifferentiated cancer cells (11-13). Recent studies indicated that BRAF likely affects the occurrence and development of thyroid cancer through the expression of other oncogenes (11).

There are different points of view regarding BRAF mutations. For patients with thyroid cancer induced by nuclear radiation, RET/PTC rearrangements, especially RET/PTC3, were significantly increased (13). However, BRAF is likely not a radiosensitive gene. Nikiforova et al (12) demonstrated that the BRAF mutation rate of patients with PCT induced by the Chernobyl nuclear accident in Ukraine was significantly lower than in sporadic PTC patients. Another study suggested that the relatively low BRAF mutation rate was associated with the young age of subjects selected in that study (13). BRAF mutation leading to tumorigenesis likely requires a longer, more latent process compared with RET/PTC rearrangement. It has also been suggested that BRAF mutation was likely associated with high iodine intake (14) and high thyroid stimulating hormone level (15). BRAF not only induces tumorigenesis, but plays an important role in tumor development and evolution. In addition, it is closely related to the clinicopathological characteristics of tumors, which is another area of great interest. Lee et al concluded that the BRAF mutation rate was $49 \%$ according to a statistical analysis of 1168 PTC patients from 12 units (16). Nikiforova et al indicated that the BRAF V600E gene mutation was found in $11.1 \%$ of cases of poorly differentiated thyroid cancer and anaplastic thyroid cancer (ATC), while the nipple structure was found in cancer tissue, suggesting that part of the ATC likely evolved from well-differentiated thyroid cancer (17). BRAF V600E gene mutation and tumor

Table II. Comparison of thyroid ultrasound features between patients with and without BRAF V600E mutations.

\begin{tabular}{|c|c|c|c|c|c|c|}
\hline \multirow[b]{2}{*}{ BRAF V600E } & \multirow[b]{2}{*}{ No. of cases } & \multirow[b]{2}{*}{ Tumor size } & \multicolumn{2}{|c|}{ Tumor border } & \multicolumn{2}{|c|}{ Calcification } \\
\hline & & & Clear & Less clear & Yes & No \\
\hline Mutation & 18 & $19.33 \pm 8.32$ & 7 & 11 & 14 & 4 \\
\hline No mutation & 16 & $17.32 \pm 7.32$ & 4 & 12 & 13 & 3 \\
\hline t-test & - & 0.63 & & & & \\
\hline P-value & - & $>0.05$ & \multicolumn{2}{|c|}{$0.487^{\mathrm{a}}$} & \multicolumn{2}{|c|}{$1.000^{\mathrm{a}}$} \\
\hline
\end{tabular}

${ }^{a}$ Exact probability test. 
prognosis is another popular topic of study. Kim et al reported that BRAF V600E mutation in the primary lesion could predict the lymph node metastasis of PTC, and its predictive value was higher than that of other clinicopathological factors such as age, clinical stage, and tumor size (18). However, the study by Costa et al suggested that it could not clearly predict the poor prognosis of PTC unless BRAF mutation and other genetic changes existed simultaneously (19). The data obtained by Xing et al from 219 multicenter studies indicated that there was a significant correlation between BRAF mutations in PTC and the following events: the invasion of cancer tissue into surrounding tissue at the time of the first operation, cervical lymph node metastasis, and thyroid cancer stage III-IV (20). Elisei et al showed that BRAF V600E was the only independent factor for predicting poor prognosis by postoperative 15-year follow-up (21). Multivariate analysis revealed that only BRAF V600E mutation and cervical lymph node metastasis were independent prognostic factors for the postoperative recurrence and metastasis of PTC. The BRAF T1799A gene mutation likely plays an important role in the growth, invasion, and dedifferentiation processes of differentiated thyroid cancer. However, factors including the number of cases, PTC subtype, clinical stage, and experimental method likely affected the detection results of the BRAF T1799A mutation.

Thus far, the BRAF V600E mutation rates reported from different studies are varied (6-8), ranging from 28 to $83 \%$, which is primarily related to epidemiological factors such as different geographical and ethnic backgrounds. In addition, some studies suggested it was related to the subtype distribution of PTC. However, BRAF V600E has not been identified in other thyroid benign or malignant tumors except for PTC, which contributes to the diagnosis and differential diagnosis of thyroid carcinoma. In the present study, the BRAF V600E mutation was detected in $52.9 \%$ of cases of PTC, which was consistent with previous studies. Detecting BRAF V600E, as a genetic test for PTC, can be combined with ultrasound-guided fine-needle aspiration (FNA) cytology. Studies suggested that detection of the BRAF T1799A mutation in cells obtained by FNA contributed to the diagnosis and differential diagnosis of PTC (22-25). With the rapid development of ultrasound technology, the advent of high resolution color Doppler ultrasound instruments, and improvement of the diagnostic level of thyroid tumors, ultrasound has become an important method for diagnosing thyroid tumors that is increasingly valuable to clinicians. To improve the differential diagnosis of thyroid benign and malignant tumors, numerous relevant studies have been carried out, and a series of diagnostic standards have been proposed. Ultrasound examination and the BRAF V600E test are two different methods for the diagnosis of thyroid cancer, and determining the association between them was one of the objectives of this study. Hwang et al suggested that in PTC patients with BRAF V600E mutation, thyroid ultrasound features had a tendency to have an anteroposterior diameter greater than the diameter (26). The incidence of calcification was also lower compared with patients without BRAF V600E mutation, but the difference was not statistically significant. In addition, there were no significant differences in nodule size, border, or echo. In the present study, we focused on tumor size, with clear or less clear border, and with or without calcification, and compared the data with the ultrasound features in patients with or without BRAF V600E mutations. There were no significant differences in ultrasound features between the two groups of patients. Therefore, ultrasound is unable to predict the presence or absence of BRAF V600E mutations. Ultrasound and BRAF V600E test are two different methods for the diagnosis and differential diagnosis of PTC, and should be analyzed in combination. The present study had limitations. The sample size was small, and the standards of thyroid ultrasound examination were not sufficiently detailed because of limitations of technological conditions. These factors restricted the study to some degree and require further investigation.

In conclusion, the BRAF V600E mutation rate is relatively high in PTC. There is no significant association between BRAF V600E mutation and thyroid ultrasound features. Thyroid ultrasound is therefore unable to predict the presence of BRAF V600E mutations in patients with PTC.

\section{References}

1. Xing M: BRAF mutation in thyroid cancer. Endocr Relat Cancer 12: 245-262, 2005.

2. Fukushima T, Suzuki S, Mashiko M, Ohtake T, Endo Y, Takebayashi Y, Sekikawa K, Hagiwara K and Takenoshita S: BRAF mutations in papillary carcinomas of the thyroid. Oncogene 22: 6455-6457, 2003.

3. Xu X, Quiros RM, Gattuso P, Ain KB and Prinz RA: High prevalence of BRAF gene mutation in papillary thyroid carcinomas and thyroid tumor cell lines. Cancer Res 63: 4561-4567, 2003.

4. Ikawa S, Fukui M, Ueyama Y, Tamaoki N, Yamamoto T and Toyoshima K: B-raf, a new member of the raf family, is activated by DNA rearrangement. Mol Cell Biol 8: 2651-2654, 1988.

5. Davies H, Bignell GR, Cox C, Stephens P, Edkins S, Clegg S, Teague J, Woffendin H, Garnett MJ, Bottomley W, et al: Mutations of the BRAF gene in human cancer. Nature 417: 949-954, 2002.

6. Cohen Y, Xing M, Mambo E, Guo Z, Wu G, Trink B, Beller U, Westra WH, Ladenson PW and Sidransky D: BR AF mutation in papillary thyroid carcinoma. J Natl Cancer Inst 95: 625-627, 2003.

7. Fugazzola L, Mannavola D, Cirello V, Vannucchi G, Muzza M, Vicentini L and Beck-Peccoz P: BRAF mutations in an Italian cohort of thyroid cancers. Clin Endocrinol (Oxf) 61: 239-243, 2004.

8. Kim KH, Kang DW, Kim SH, Seong IO and Kang DY: Mutations of the BRAF gene in papillary thyroid carcinoma in a Korean population. Yonsei Med J 45: 818-821, 2004.

9. Sithanandam G, Druck T, Cannizzaro LA, Leuzzi G, Huebner K and Rapp UR: B-raf and a B-raf pseudogene are located on 7q in man. Oncogene 7: 795-799, 1992.

10. Mercer KE and Pritchard CA: Raf proteins and cancer: B-Raf is identified as a mutational target. Biochim Biophys Acta 1653: 25-40, 2003.

11. Soares P, Trovisco V, Rocha AS, Lima J, Castro P, Preto A, Máximo V, Botelho T, Seruca R and Sobrinho-Simões M: BRAF mutations and RET/PTC rearrangements are alternative events in the etiopathogenesis of PTC. Oncogene 22: 4578-4580, 2003.

12. Nikiforova MN, Ciampi R, Salvatore G, Santoro M, Gandhi M, Knauf JA, Thomas GA, Jeremiah S, Bogdanova TI, Tronko MD, et al: Low prevalence of BRAF mutations in radiation-induced thyroid tumors in contrast to sporadic papillary carcinomas. Cancer Lett 209: 1-6, 2004.

13. Lima J, Trovisco V, Soares P, Máximo V, Magalhães J, Salvatore G, Santoro M, Bogdanova T, Tronko M, Abrosimov A, et al: Reply to: Low prevalence of BRAF mutations in radiation-induced thyroid tumors in contrast to sporadic papillary carcinomas. Cancer Lett 230: 149-150, 2005.

14. Guan H, Ji M, Bao R, Yu H, Wang Y, Hou P, Zhang Y, Shan Z, Teng $\mathrm{W}$ and Xing $\mathrm{M}$ : Association of high iodine intake with the T1799A BRAF mutation in papillary thyroid cancer. J Clin Endocrinol Metab 94: 1612-1617, 2009. 
15. Watanabe R, Hayashi Y, Sassa M, Kikumori T, Imai T, Kiuchi T and Murata Y: Possible involvement of BRAF V600E in altered gene expression in papillary thyroid cancer. Endocr J 56: 407-414, 2009.

16. Lee JH, Lee ES and Kim YS: Clinicopathologic significance of BRAF V600E mutation in papillary carcinomas of the thyroid: A meta-analysis. Cancer 110: 38-46, 2007.

17. Nikiforova MN, Kimura ET, Gandhi M, Biddinger PW, Knauf JA, Basolo F, Zhu Z, Giannini R, Salvatore G, Fusco A, et al: BRAF mutations in thyroid tumors are restricted to papillary carcinomas and anaplastic or poorly differentiated carcinomas arising from papillary carcinomas. J Clin Endocrinol Metab 88: 5399-5404, 2003.

18. Kim J, Giuliano AE, Turner RR, Gaffney RE, Umetani N, Kitago M, Elashoff D and Hoon DS: Lymphatic mapping establishes the role of BRAF gene mutation in papillary thyroid carcinoma. Ann Surg 244: 799-804, 2006.

19. Costa AM, Herrero A, Fresno MF, Heymann J, Alvarez JA, Cameselle-Teijeiro J and García-Rostán G: BRAF mutation associated with other genetic events identifies a subset of aggressive papillary thyroid carcinoma. Clin Endocrinol (Oxf) 68: 618-634, 2008.

20. Xing M, Westra WH, Tufano RP, Cohen Y, Rosenbaum E, Rhoden KJ, Carson KA, Vasko V, Larin A, Tallini G, et al BRAF mutation predicts a poorer clinical prognosis for papillary thyroid cancer. J Clin Endocrinol Metab 90: 6373-6379, 2005.

21. Elisei R, Ugolini C, Viola D, Lupi C, Biagini A, Giannini R, Romei C, Miccoli P, Pinchera A and Basolo F: BRAF(V600E) mutation and outcome of patients with papillary thyroid carcinoma: A 15-year median follow-up study. J Clin Endocrinol Metab 93: 3943-3949, 2008.
22. Kim DL, Song KH and Kim SK: High prevalence of carcinoma in ultrasonography-guided fine needle aspiration cytology of thyroid nodules. Endocr J 55: 135-142, 2008.

23. Xing M, Tufano RP, Tufaro AP, Basaria S, Ewertz M, Rosenbaum E, Byrne PJ, Wang J, Sidransky D and Ladenson PW: Detection of BRAF mutation on fine needle aspiration biopsy specimens: A new diagnostic tool for papillary thyroid cancer. J Clin Endocrinol Metab 89: 2867-2872, 2004.

24. Chung KW, Yang SK, Lee GK, Kim EY, Kwon S, Lee SH, Park DJ, Lee HS, Cho BY, Lee ES, et al: Detection of BRAF V600E mutation on fine needle aspiration specimens of thyroid nodule refines cyto-pathology diagnosis, especially in BRAF600E mutation-prevalent area. Clin Endocrinol (Oxf) 65: 660-666, 2006

25. Kumagai A, Namba H, Akanov Z, Saenko VA, Meirmanov S, Ohtsuru A, Yano H, Maeda S, Anami M, Hayashi T, et al: Clinical implications of pre-operative rapid BRAF analysis for papillary thyroid cancer. Endocr J 54: 399-405, 2007.

26. Hwang J, Shin JH, Han BK, Ko EY, Kang SS, Kim JW and Chung JH: Papillary thyroid carcinoma with BRAF V600E mutation: Sonographic prediction. AJR Am J Roentgenol 194: W425-W430, 2010. 